\title{
Relação entre problemas do sono, desempenho funcional e ocorrência de quedas em idosos da comunidade
}

Relationship between sleep problems, functional performance and falls in community-dwelling elderly

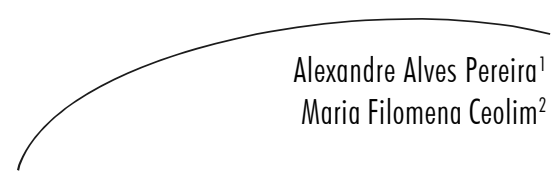

Resumo

Objetivo: Esta revisão objetivou sistematizar estudos que avaliam a relação entre problemas do sono, desempenho funcional e ocorrência de quedas em idosos da comunidade. Método: Realizou-se revisão bibliográfica entre 21 e 30 de setembro de 2009, no idioma Inglês, com as seguintes palavras-chave: "sleep" AND "falls" AND "functional performance" AND "elderly". Foram utilizadas as bases de dados PubMed, Ageline e Scielo. Foram critérios de inclusão: artigos publicados de janeiro de 2006 a setembro de 2009; artigos completos; estudos transversais, retrospectivos, prospectivos; estudos realizados com idosos da comunidade. Resultados: Foram encontrados 52 estudos, dos quais foram avaliados dez que preencheram os critérios de inclusão. Conclusões: Os estudos mostraram que os distúrbios do sono podem piorar o desempenho funcional, além de representarem fator de risco independente para quedas em idosos.

\section{Abstract}

Objective: This study aimed to systematize the studies that examined the relationship between sleep problems, functional performance and falls in community-dwelling elderly. Methods: We performed a literature review in English from 21 to 30 September 2009, using the key words: "sleep" AND "falls" AND "functional performance" AND "elderly". We used the databases PubMed, Ageline and Scielo. Inclusion criteria were: articles published from January 2006 to September 2009; full texts; cross-sectional design, retrospective, prospective; studies performed with community-dwelling elderly. Results: We found 52 studies, of which 10 were assessed by the inclusion criteria. Conclusions: Studies show that sleep problems may worsen the functional performance, as well as be an independent factor for falls in community-dwelling elderly.

\footnotetext{
1 Programa de Pós-graduação em Gerontologia. Faculdade de Ciências Médicas. Universidade Estadual de Campinas - Unicamp. Campinas, SP, Brasil.

2 Departamento de Enfermagem. Faculdade de Ciências Médicas. Universidade Estadual de Campinas - Unicamp. Campinas, SP. Brasil
}

Palavras-chave: Idoso.
Saúde do idoso. Acidentes
por quedas. Privação do
sono. Transtorno do sono.
Avaliação de desempenho.

Key words: Aged. Health of the Elderly. Accidental Falls. Sleep Deprivation. Sleep Disorders. Employee Performance Appraisal. 


\section{INTRODUÇÃO}

A carga de doenças relacionadas aos agravos não-transmissíveis tem se elevado rapidamente e sua prevenção tem sido o maior desafio para a saúde pública. ${ }^{1}$ Nessa perspectiva, as quedas de idosos são atualmente uma das preocupações por sua frequência e por trazerem consequências negativas à qualidade de vida dessas pessoas..$^{2-5}$

As quedas em idosos possuem significado muito relevante, pois podem levá-los à incapacidade, injúria e morte; seu custo social é elevado e torna-se maior quando o idoso tem diminuição da autonomia e da independência, ou passa a necessitar de institucionalização. . $^{3,6-8}$

Estudo transversal, com amostra composta por 4.003 idosos (65 anos ou mais) residentes na área de abrangência de unidades básicas de saúde de 41 municípios com mais de 100 mil habitantes, de sete estados brasileiros, mostrou que a prevalência de quedas entre idosos foi de $34,8 \%$, sendo significativamente maior em mulheres (40,1\%); a prevalência de quedas associou-se com idade avançada, sedentarismo, autopercepção da saúde sendo ruim e referência a maior número de medicações de uso contínuo. ${ }^{9}$

No Brasil, em 2000, as quedas foram responsáveis por 2.030 mortes na faixa de 60 anos ou mais, ocupando o terceiro lugar na mortalidade por causas externas, tanto entre homens quanto entre mulheres. Em relação à morbidade, as quedas aumentam sua importância, ocupando o primeiro lugar nas internações. Neste mesmo ano, 48.940 pessoas foram hospitalizadas devido à ocorrência de quedas. Em relação às lesões determinadas por esses acidentes, $68,3 \%$ são fraturas, chamando a atenção as fraturas de fêmur. ${ }^{10}$

Considerando-se sua natureza etiológica múltipla, é importante compreender os fatores relacionados a quedas e traçar o perfil de ocorrência das mesmas, procurando investigar as variáveis envolvidas, uma vez que a eficácia de medidas para prevenção de quedas depende da identificação das variáveis relacionadas com risco de quedas em idosos.
Além das variáveis de saúde, de capacidade funcional e relacionadas ao contexto ambiental, , ,11 outro fator de risco tem sido mencionado na literatura. ${ }^{12}$ Trata-se de problemas do sono que, por sua vez, são associados às mudanças fisiológicas do envelhecimento normal, à presença de comorbidades, à depressão e ao uso de medicamentos, entre outros. ${ }^{13}$ Há poucos estudos avaliando a relação entre distúrbios do sono no envelhecimento e sua relação com a ocorrência de quedas em indivíduos idosos. Entretanto, vem aumentando o interesse pela compreensão do fenômeno do sono na pessoa idosa, com o intuito de melhorar a qualidade de vida desta população. ${ }^{14}$

O sono é um processo fisiológico vital. São caracterizados dois padrões fundamentais de sono: sem movimentos oculares rápidos (NREM, do inglês Non-rapid eyes moviment) e com movimentos oculares rápidos (REM). ${ }^{15,16} \mathrm{O}$ sono NREM é composto por quatro etapas em grau crescente de profundidade: os estágios I e II, superficiais, e os estágios profundos III e IV, ou sono delta. No sono NREM há relaxamento muscular comparativamente à vigília, porém é mantida alguma tonicidade basal. O sono REM ou sono paradoxal, embora seja um estágio profundo no tocante à dificuldade de despertar, o indivíduo nessa fase exibe padrão eletrencefalográfico que se assemelha à vigília, sendo este um dos seus aparentes paradoxos. Além disso, apesar da atonia muscular que acompanha este estágio, observamse movimentos corporais fásicos e erráticos, de diversos grupamentos musculares, principalmente na face e nos membros, bem como emissão de sons. Ou seja, mesmo em meio à inibição motora, há liberação fásica de atividade muscular de localização multifocal.

Quanto à arquitetura do sono, em condições normais um indivíduo inicia o sono noturno pelo estágio I do sono NREM, após uma latência aproximada de 10 minutos. Seguindo-se uns poucos minutos no estágio I, ocorre o aprofundamento para o estágio II. Depois de 30 a 60 minutos, instala-se o sono delta. Aproximadamente 90 minutos após o início do sono, acontece o primeiro sono REM, que 
costuma ter curta duração no início da noite (5 a 10 minutos), completando-se o primeiro ciclo NREM-REM. A saída do sono REM para os estágios superficiais do sono NREM pode ocorrer com intrusão de microdespertar ( 3 a 15 segundos de duração), sem um despertar completo do indivíduo. Desta forma, cumprem-se cerca de cinco a seis ciclos de sono NREM-REM, durante uma noite de oito horas de sono. ${ }^{15,16}$

A velhice, por si só, não é causa direta de problemas relacionados ao sono, ${ }^{13,17}$ embora torne o idoso mais vulnerável a essas ocorrências. ${ }^{18} \mathrm{Com}$ o envelhecimento, ocorrem mudanças quantitativas e qualitativas do sono. ${ }^{19,20} \mathrm{O}$ tempo total de sono noturno diminui significantemente com o avançar da idade, porém tende a manterse após os 65 anos. ${ }^{19,21,22} \mathrm{~A}$ principal mudança ocorre na arquitetura do sono, com a redução percentual do sono de ondas lentas e REM. ${ }^{21} \mathrm{O}$ estágio IV não mais se registra, havendo redução do estágio III e aumento dos despertares noturnos, ${ }^{15}$ o que traz como consequência direta sua menor eficiência, principalmente na presença de comorbidades, e do uso de medicamentos para tratar essas condições. ${ }^{23,24}$ As principais doenças relacionadas à má qualidade do sono em idosos são: depressão, alterações de humor e ansiedade, cardiopatia, diabetes, doença pulmonar obstrutiva crônica, dor crônica, doença gastrointestinal, Alzheimer e doença de Parkinson. ${ }^{24-26}$

A insônia é a queixa de alteração do sono mais comum entre idosos. ${ }^{26}$ De acordo com o DSM IV $^{27}$ (Diagnostic and Statistical Manual of Mental Disorders - IV), a insônia é definida como uma queixa de insatisfação com a qualidade e quantidade do sono, composta por um ou mais dos seguintes sintomas: dificuldade em iniciar o sono, dificuldade em manter o sono, que se caracteriza por frequentes despertares noturnos ou dificuldade em retomar o sono após despertar, despertar precoce e sono não restaurador. Essas queixas, coletivamente denominadas de problemas do sono, são acompanhadas por prejuízo no desempenho de atividades diurnas, indicado por pelo menos um dos seguintes sintomas: fadiga ou baixa energia, sonolência diurna, déficits de atenção, concentração e memória, irritabilidade, hiperatividade e agressão, diminuição no rendimento profissional e nas relações sociais. ${ }^{28,29}$

A insônia pode ser classificada como autoinduzida, secundária ou primária. Os fatores relacionados à insônia autoinduzida são estilo de vida (estresse e fatores ambientais) e interrupção abrupta ou uso abusivo de substâncias psicoativas (álcool, cafeína, hipnóticos, ansiolíticos, sedativos, anfetaminas e opióides); quanto à insônia secundária, as principais relações referem-se aos distúrbios do humor (depressão, ansiedade), doenças (artrite, cardiopatia, doença de Parkinson), distúrbios respiratórios durante o sono (apneia do sono) e outros distúrbios do sono (síndrome das pernas inquietas, distúrbios do ritmo circadiano); quanto à insônia primária, ainda não foram identificados os fatores causais relacionados à sua ocorrência. ${ }^{29}$ Desta forma, a insônia parece representar a queixa maior de alterações do sono, e envolve um grupo vasto de fatores causais. ${ }^{30,31} \mathrm{Em}$ idosos nãoinstitucionalizados, a dificuldade em iniciar o sono foi relatada por 15 a $45 \%$ da população; sono fragmentado, em aproximadamente 20 a 65\%; despertar precoce em 15 a 54\%; e cansaço após noite de sono em aproximadamente $10 \%$ da população estudada. O estudo também afirma que a prevalência de insônia é maior em mulheres do que em homens. ${ }^{29}$

As consequências clínicas dos problemas do sono em idosos podem resultar frequentemente em desorientação, alteração cognitiva, retardo psicomotor e diminuição da função física, com implicações diretas nas atividades de vida diária (AVDs), risco aumentado de acidentes e traumas, pior estado de saúde e aumento do risco de morte. ${ }^{24,31}$ Há uma associação direta entre noites de sono de má qualidade e o desempenho funcional diurno. ${ }^{26}$ Desta forma, sugere-se que a má qualidade do sono constitui fator independente de risco de queda em idosos. ${ }^{12}$ Sendo assim, buscou-se com esta revisão sistematizar os estudos que examinaram a relação entre distúrbios do sono, desempenho funcional e ocorrência de quedas em idosos da comunidade. 


\section{MÉTODOS}

Foi realizada revisão bibliográfica entre 21 e 30 de setembro de 2009, no idioma inglês, com as seguintes palavras-chave: "sleep" AND "falls" AND "functional performance" AND "elderly". As bases de dados PubMed, Ageline e Scielo foram consultadas, e os critérios de inclusão foram: artigos publicados de janeiro de 2006 a setembro de 2009; artigos completos; estudos transversais, retrospectivos, prospectivos; estudos realizados com idosos da comunidade.

\section{RESULTADOS}

Foram encontrados 52 estudos, dos quais foram avaliados dez, por preencherem os critérios de inclusão. Cinco estudos foram realizados nos Estados Unidos da América (EUA), ${ }^{32-36}$ quatro na Austrália, ${ }^{37-40}$ e um em Taiwan. ${ }^{41} \mathrm{O}$ número de sujeitos nas amostras variou de 247 a 8.101 (quadro 1).

Quadro1- Principais achados de 10 estudos publicados entre 2006 e 2009 relativos à associação entre distúrbios do sono, desempenho funcional e ocorrência de quedas em idosos da comunidade. Campinas, SP, 2009.

\begin{tabular}{|c|c|c|c|c|}
\hline $\begin{array}{c}\text { Autor / Ano } \\
\text { / Local }\end{array}$ & Objetivo & Amostra & Delineamento & Resultados e conclusões \\
\hline $\begin{array}{l}\text { Stone et al, } \\
2006 ;{ }^{34} \text { EUA }\end{array}$ & $\begin{array}{l}\text { Avaliar a associação } \\
\text { entre sono, cochilos } \\
\text { diurnos auto-relatados } \\
\text { e risco de queda e } \\
\text { fraturas em idosas da } \\
\text { comunidade }\end{array}$ & $\begin{array}{l}8.101 \text { mulheres com } \\
69 \text { anos ou mais }\end{array}$ & $\begin{array}{l}\text { Estudo } \\
\text { Transversal }\end{array}$ & $\begin{array}{l}\text { Idosas que relataram } 10 \\
\text { horas ou mais de sono nas } \\
24 \text { horas e cochilos diurnos } \\
\text { acima de } 3 \text { horas por } \\
\text { semana apresentaram } \\
\text { probabilidade aumentada } \\
\text { de duas ou mais quedas } \\
\text { (OR }=1,50 \text { e } 1,33 \\
\text { respectivamente) }\end{array}$ \\
\hline $\begin{array}{l}\text { Goldman et } \\
\text { al, 2007; } \\
\text { EUA }\end{array}$ & $\begin{array}{l}\text { Avaliar a associação } \\
\text { entre distúrbios do } \\
\text { sono e desempenho } \\
\text { funcional em idosas da } \\
\text { comunidade }\end{array}$ & $\begin{array}{l}2.889 \text { mulheres }(83.5 \\
\pm 3,7 \text { anos) }\end{array}$ & $\begin{array}{l}\text { Estudo } \\
\text { Transversal }\end{array}$ & $\begin{array}{l}\text { As mulheres que dormiam } \\
\text { menos de seis horas por } \\
\text { noite apresentaram a } \\
\text { velocidade da marcha } 3,5 \% \\
\text { mais lenta do que aqueles } \\
\text { que dormiram de } 6,0 \text { a } 6,8 \\
\text { horas. Nas que } \\
\text { apresentaram maior } \\
\text { quantidade despertares } \\
\text { noturnos após o início do } \\
\text { sono ( } \geq 1,6 \text { horas } \\
\text { comparado } \leq 0,7 \text { horas) a } \\
\text { velocidade da marcha foi } \\
9,1 \% \text { mais lenta, e levaram } \\
7,6 \% \text { mais tempo para } \\
\text { realizar a tarefa de levantar } \\
\text { e sentar cinco vezes de uma } \\
\text { cadeira }\end{array}$ \\
\hline $\begin{array}{l}\text { Stone et al, } \\
2008 ;{ }^{36} \text { EUA }\end{array}$ & $\begin{array}{l}\text { Avaliar a associação } \\
\text { entre a duração do } \\
\text { sono noturno e o risco } \\
\text { de queda em idosas da } \\
\text { comunidade }\end{array}$ & $\begin{array}{l}2.978 \text { mulheres com } \\
70 \text { anos ou mais }\end{array}$ & $\begin{array}{l}\text { Estudo } \\
\text { Transversal }\end{array}$ & $\begin{array}{l}\text { Período de sono noturno } \\
\text { inferior a cinco horas } \\
\text { aumentou } \\
\text { aproximadamente uma vez } \\
\text { e meia a chance de queda } \\
\text { (OR } 1,52 \text { ), quando } \\
\text { comparado a duração do } \\
\text { sono entre sete e oito } \\
\text { horas, independente do uso } \\
\text { de benzodiazepínicos }\end{array}$ \\
\hline
\end{tabular}


Quadro 1 - Principais achados de 10 estudos publicados entre 2006 e 2009 relativos à associação entre distúrbios do sono, desempenho funcional e ocorrência de quedas em idosos da comunidade. Campinas, SP, 2009. (Continuação)

\begin{tabular}{|c|c|c|c|c|}
\hline $\begin{array}{c}\text { Autor / Ano } \\
\text { / Local }\end{array}$ & Objetivo & Amostra & Delineamento & Resultados e conclusões \\
\hline $\begin{array}{l}\text { Dam et al, } \\
2008 ;{ }^{37} \text { EUA }\end{array}$ & $\begin{array}{l}\text { Avaliar a associação } \\
\text { entre qualidade do } \\
\text { sono e desempenho } \\
\text { físico em idosos da } \\
\text { comunidade }\end{array}$ & $\begin{array}{l}2.862 \text { homens }(76,4 \\
\pm 5,5 \text { anos })\end{array}$ & $\begin{array}{l}\text { Estudo } \\
\text { Transversal }\end{array}$ & $\begin{array}{l}\text { Despertar noturno por } \\
\text { tempo superior a } 90 \\
\text { minutos esteve associado } \\
\text { com pior função física } \\
\text { entre os idosos avaliados. }\end{array}$ \\
\hline $\begin{array}{l}\text { Endeshaw, } \\
2009 ; ;^{42} \text { EUA }\end{array}$ & $\begin{array}{l}\text { Avaliar a associação } \\
\text { entre problemas do } \\
\text { sono, noctúria e queda } \\
\text { em idosos da } \\
\text { comunidade }\end{array}$ & $\begin{array}{l}103 \text { homens }(76,6 \pm \\
5.6 \text { anos) } \\
144 \text { mulheres }(75,1 \\
\pm 6.9 \text { anos) }\end{array}$ & $\begin{array}{l}\text { Estudo } \\
\text { Transversal }\end{array}$ & $\begin{array}{l}137 \text { participantes foram } \\
\text { distribuídos em três } \\
\text { grupos: os que relataram } \\
\text { noctúria }(22 \%) \text {; noctúria } \\
\text { sem dificuldade de retomar } \\
\text { o sono }(55 \%) \text { e os que } \\
\text { relataram noctúria e } \\
\text { dificuldade de voltar a } \\
\text { dormir ( } 23 \%) \text {. O último } \\
\text { grupo relatou uma ou mais } \\
\text { quedas nos seis meses } \\
\text { anteriores quando } \\
\text { comparado aos demais } \\
\text { grupos. }\end{array}$ \\
\hline $\begin{array}{l}\text { Teo et al, } \\
2006 ;^{41} \\
\text { Austrália }\end{array}$ & $\begin{array}{l}\text { Avaliar a associação } \\
\text { entre distúrbios do } \\
\text { sono, sonolência } \\
\text { diurna, incontinência } \\
\text { urinária e risco de } \\
\text { queda em idosos da } \\
\text { comunidade }\end{array}$ & $\begin{array}{l}782 \text { mulheres: } 75- \\
86 \text { anos }\end{array}$ & $\begin{array}{l}\text { Estudo } \\
\text { Transversal }\end{array}$ & $\begin{array}{l}\text { Incontinência urinária e } \\
\text { sonolência diurna excessiva } \\
\text { foram fatores de risco } \\
\text { independentes de queda } \\
\text { (OR } 1,76 \text { e } 2,05 \\
\text { respectivamente) após o } \\
\text { controle estatístico de } \\
\text { outros fatores de risco de } \\
\text { queda (idade, uso de } \\
\text { sedativos e uso de drogas } \\
\text { cardiovasculares). }\end{array}$ \\
\hline $\begin{array}{l}\text { St George et } \\
\text { al, 2008; }{ }^{38} \\
\text { Austrália }\end{array}$ & $\begin{array}{l}\text { Avaliar a associação } \\
\text { entre qualidade do } \\
\text { sono, cochilos diurnos } \\
\text { e queda em idosos da } \\
\text { comunidade }\end{array}$ & $\begin{array}{l}491 \text { mulheres e } 81 \\
\text { homens }(79,4 \pm 6,4 \\
\text { anos) }\end{array}$ & $\begin{array}{l}\text { Estudo } \\
\text { Transversal }\end{array}$ & $\begin{array}{l}\text { Participantes que relataram } \\
\text { cochilos maiores que } 30 \\
\text { minutos por dia e menos } \\
\text { de } 6 \text { horas de sono por } \\
\text { noite apresentaram três } \\
\text { vezes mais chances de cair } \\
\text { (OR } 3,13 \text { e } 3,10 \\
\text { respectivamente) após } \\
\text { ajuste por idade, gênero, } \\
\text { uso de psicotrópicos, tipo } \\
\text { de acomodação para } \\
\text { dormir e estado de saúde. }\end{array}$ \\
\hline $\begin{array}{l}\text { Hill et al, } \\
2007 ;^{32} \\
\text { Austrália }\end{array}$ & $\begin{array}{l}\text { Avaliar a associação } \\
\text { entre distúrbios do } \\
\text { sono e queda em idosos } \\
\text { institucionalizados e da } \\
\text { comunidade }\end{array}$ & $\begin{array}{l}150 \text { idosos } \\
\text { institucionalizados } \\
(81 \pm 8 \text { anos); } 150 \\
\text { idosos da } \\
\text { comunidade usuários } \\
\text { da internet ( } 70 \pm 5 \\
\text { anos) }\end{array}$ & $\begin{array}{l}\text { Estudo } \\
\text { Transversal }\end{array}$ & $\begin{array}{l}\text { Alterações do sono foram } \\
\text { comuns nos idosos da } \\
\text { amostra. Entre os idosos } \\
\text { institucionalizados as } \\
\text { quedas foram associadas à } \\
\text { má qualidade do sono (OR } \\
\text { 4,5) e fragmentação do } \\
\text { sono noturno (OR 2,5). }\end{array}$ \\
\hline
\end{tabular}




\begin{tabular}{|c|c|c|c|c|}
\hline $\begin{array}{c}\text { Autor / Ano } \\
\text { / Local }\end{array}$ & Objetivo & Amostra & Delineamento & Resultados e conclusões \\
\hline & & & & $\begin{array}{l}\text { Entre os idosos da } \\
\text { comunidade não foi } \\
\text { encontrada associação entre } \\
\text { uso de hipnótico, qualidade } \\
\text { do sono e risco de queda. }\end{array}$ \\
\hline $\begin{array}{l}\text { Kaushik et } \\
\text { al, 2007;33 } \\
\text { Austrália }\end{array}$ & $\begin{array}{l}\text { Avaliar a associação } \\
\text { entre eficiência do } \\
\text { sono e quedas em } \\
\text { idosos da comunidade }\end{array}$ & $\begin{array}{l}\text { 1.952 participantes: } \\
60-97 \text { anos }\end{array}$ & $\begin{array}{l}\text { Estudo } \\
\text { Transversal }\end{array}$ & $\begin{array}{l}\text { Idosos com apneia do sono } \\
\text { apresentaram risco } \\
\text { aumentado de queda em } \\
\text { duas ou mais vezes (OR } \\
2,23) \text { quando comparados } \\
\text { aos idosos sem apneia, após } \\
\text { o controle das variáveis } \\
\text { associadas à queda. }\end{array}$ \\
\hline $\begin{array}{l}\text { Kuo et al, } \\
2009 ;^{39} \\
\text { Taiwan }\end{array}$ & $\begin{array}{l}\text { Avaliar a associação } \\
\text { entre duração do sono } \\
\text { e quedas em idosos da } \\
\text { comunidade }\end{array}$ & $\begin{array}{l}135 \text { mulheres e } 121 \\
\text { homens com média } \\
\text { de idade de } 72,2 \text { anos }\end{array}$ & $\begin{array}{l}\text { Estudo } \\
\text { Transversal }\end{array}$ & $\begin{array}{l}\text { Dormir menos que } 5 \text { horas } \\
\text { por noite esteve associado a } \\
\text { um risco aumentado de } \\
\text { queda em mulheres (OR } \\
2,98 \text { ) quando comparadas } \\
\text { ao grupo de mulheres que } \\
\text { dormiam acima de cinco } \\
\text { horas noturnas; esta } \\
\text { associação não foi } \\
\text { encontrada entre os } \\
\text { homens da amostra. }\end{array}$ \\
\hline
\end{tabular}

OR: odds ratio.

\section{DISCUSSÃO}

O risco aumentado de queda em idosos é uma das principais consequências clínicas dos problemas do sono nessa faixa etária. ${ }^{26} \mathrm{Num}$ estudo prospectivo cujo objetivo foi avaliar a prevalência de preditores de queda em uma amostra de idosos da comunidade, com 65 anos ou mais, os distúrbios do sono foram evidenciados como fatores de risco de queda. ${ }^{2}$ Semelhantemente, Ribeiro et $\mathrm{al}^{5}$ encontraram significativa associação entre distúrbios do sono e queda, caracterizando-os como mais um fator intrínseco de risco de queda em idosos.

Em um estudo transversal, ${ }^{37}$ com 150 idosos institucionalizados e 150 idosos da comunidade, evidenciou-se que a ocorrência de quedas no primeiro grupo esteve associada à má qualidade do sono, despertar noturno, uso de diuréticos, dor e depressão. No grupo da comunidade, quedas estiveram associadas a doenças, déficit visual, uso de óculos bifocais e uso de órteses para o auxílio da marcha. Nesta perspectiva, pesquisa realizada com uma amostra de 1.952 idosos, após o controle dos fatores de risco de queda, mostrou que aqueles que tinham diagnóstico de apneia do sono apresentavam risco duas vezes maior de cair, quando comparados aos que não apresentavam este distúrbio do sono. ${ }^{38}$

Os mecanismos fisiológicos envolvidos ainda são pouco esclarecidos. Possivelmente, são multifatoriais e incluem a diminuição do equilíbrio e do tempo de reação postural, e o efeito residual dos medicamentos usados para tratar os problemas do sono, caracterizando uma situação em que tanto os problemas do sono por si só, quanto os medicamentos usados para tratálos podem contribuir para o risco de queda. ${ }^{26}$

No estudo multicêntrico SOF (Study of Osteoporotic Fractures), 32 os pesquisadores investigaram a relação entre autorrelato de qualidade do sono e cochilos durante o dia, e a ocorrência de queda e fraturas numa amostra de 
8.101 mulheres. Os achados mostraram, após ajuste multivariado, que mulheres que cochilavam durante o dia, pelo menos três horas por semana, tinham duas ou três vezes mais chances de cair e sofreram mais fraturas do que mulheres que cochilavam menos que três horas. Em relação ao tempo total de sono nas 24 horas, após ajuste por idade, os pesquisadores encontraram que as mulheres que informavam um tempo total acima de dez horas de sono tiveram duas ou mais chances de cair, quando comparadas às que informavam um tempo total de sono em torno de oito horas diárias. O mesmo grupo de pesquisadores investigou a relação entre problemas do sono e desempenho funcional, numa amostra de 2.889 mulheres com média de idade de 83.5 anos. Os resultados encontrados sugerem que mulheres que apresentaram má qualidade do sono, tiveram pior desempenho funcional nas atividades de vida diária. ${ }^{33}$

Ainda no Estudo SOF, ${ }^{34}$ em 2008, os investigadores coletaram dados de actimetria usada por cinco dias, em uma amostra de 2.978 mulheres de 70 anos ou mais (média de 84 anos); após a coleta, as idosas foram acompanhadas por um ano para relato de possíveis quedas e fraturas. Outras medidas coletadas foram: altura, peso, Índice de Massa Corpórea (IMC), força de preensão, velocidade da marcha, levantar-se de uma cadeira cinco vezes sem ajuda dos braços ou auxílio de outra pessoa, uso de medicamentos (incluindo benzodiazepínicos, antipsicóticos e antidepressivos), sintomas depressivos, ansiedade, status cognitivo, atividades instrumentais de vida diária (AIVD), atividade física, consumo de álcool, doenças diagnosticadas e sonolência diurna. Após ajuste por idade e raça, observou-se relação entre horas de sono e risco de queda, ou seja, o risco era maior para aquelas que dormiam menos de cinco horas por noite, assim como para as que dormiam mais que oito horas noturnas.

Verificou-se também relação entre insatisfação e fragmentação do sono, com o risco aumentado de queda. Ajustes multivariados mostraram que poucas horas de sono e o despertar repetitivo acima de 120 minutos por noite têm relação com risco de queda, independentemente do uso de benzodiazepínicos, IMC ou comorbidades. Aliás, o estudo mostrou que não houve relação significativa entre uso de benzodiazepínicos e risco de quedas. Os pesquisadores sugerem, inclusive, que o período de sono curto poderia ser considerado um marcador de fragilidade entre idosos, já que tem relação com déficit de desempenho funcional e risco de queda.

O Osteoporotic Fractures in Men Study (MrOS) ${ }^{35}$ evidenciou resultados semelhantes. Com uma amostra de 2.862 homens acima de 67 anos, avaliados através de polissonografia e actigrafia, o estudo indicou que má qualidade do sono, despertar noturno por mais de 90 minutos e eficiência do sono menor que $80 \%$ estiveram associados à menor força de preensão, menor velocidade da marcha, incapacidade de levantarse de uma cadeira sem assistência, e inabilidade em percorrer pequenos trajetos após ajustes por idade, IMC, uso de antidepressivos, hipertensão arterial, outras comorbidades (história de pelo menos uma condição médica, incluindo doenças cardiovasculares, osteoartrite, diabetes melittus e doença de Parkinson), escala de atividade física para idosos, e fumo. Os autores concluíram que o sono fragmentado e hipóxia durante a noite estão relacionados com redução de desempenho físico durante o dia.

No estudo conduzido por Faubel et al., ${ }^{14} \mathrm{em}$ comparação com as idosas que dormiam sete horas, aquelas que dormiam menos de cinco ou mais de dez horas apresentavam piores escores de qualidade de vida. Similarmente, St George et al., ${ }^{39}$ com uma amostra de 572 idosos, com média de idade de 79,9 anos, mostraram que aqueles que cochilavam mais do que 30 minutos por dia e dormiam menos que seis horas por noite tinham três vezes mais chances de cair, controlados os demais fatores de risco de queda. Da mesma forma, pesquisadores em Taiwan, com uma amostra de 256 idosos (135 mulheres e 121 homens), cujo objetivo foi avaliar a associação entre gênero, horas de sono noturno e queda em idosos, encontraram importante associação entre dormir menos que cinco horas noturnas e a ocorrência de queda, principalmente entre as mulheres. ${ }^{41}$ 
Outro fator que tem sido relacionado à alteração do sono, e ocorrência de queda em idosos é a noctúria. ${ }^{42}$ A poliúria, uma das causas de noctúria, associa-se a algumas doenças, tais como diabetes melittus e insuficiência cardíaca congestiva; interfere diretamente na qualidade do sono e acarreta sonolência diurna, prejudicando o desempenho funcional de idosos. ${ }^{42}$ Pesquisadores australianos examinaram o efeito independente de autorrelato de características do sono e incontinência urinária em relação ao risco de queda, em uma amostra de 786 mulheres acima de 70 anos. Os achados revelaram que sonolência diurna excessiva esteve associada a um risco duas vezes maior de queda, após o controle de fatores de risco associados, incluindo incontinência urinária e uso de benzodiazepínicos. ${ }^{40}$ Nesta mesma perspectiva, outro estudo mostrou que noctúria, uso de benzodiazepínicos e má qualidade do sono são fatores independentes de risco de queda em idosos; as características clínicas dos participantes do estudo com noctúria e dificuldades para voltar a dormir foram diferentes daqueles com noctúria, mas sem dificuldade para retomar o sono, sugerindo desta forma, que haveria diferentes fatores etiológicos para ambos os grupos. Os achados sugerem que muitos dos eventos relatados como noctúria podem ser precedidos por despertares noturnos não relacionados a sintomas urinários, mas a alterações primárias do sono, tais como apneia do sono ou síndrome das pernas inquietas; como as quedas relacionadas à noctúria correspondem às idas ao banheiro à noite, a ligação entre má qualidade do sono e quedas, neste caso, estaria relacionada à sonolência diurna. ${ }^{36}$

\section{CONSIDERAÇÕES FINAIS}

Problemas do sono são comuns com o avançar da idade, e ocorrem em mais da metade dos indivíduos acima dos 65 anos. Apesar da prevalência elevada, a velhice por si só não é causa de distúrbios do sono. Essas alterações comumente estão associadas à comorbidades e a piores escores de qualidade de vida.

Intervir preventivamente pressupõe a realização de investigações detalhadas e bem direcionadas. Ações preventivas baseadas em evidências claras e pontuais trazem como consequência melhor condição de vida e bemestar da população-alvo. Neste sentido, percebese que ainda se destina pouca atenção a avaliação da qualidade do sono em idosos e seus possíveis desdobramentos. Por outro lado, tanto os distúrbios do sono, quanto a ocorrência de quedas comprometem a qualidade de vida dos idosos. Investigar esta interação torna-se relevante por revelar possíveis associações ainda pouco conhecidas, cuja melhor compreensão pode trazer benefícios a esta população.

Até o presente, a maioria dos estudos que investigaram a relação entre distúrbios do sono e quedas em idosos são de caráter transversal. Entretanto, a ocorrência de quedas em idosos possui natureza etiológica multifatorial, e assim os estudos prospectivos são mais refinados para determinar e explorar os fatores de risco de quedas em idosos. As amostras de conveniência não são generalizáveis; portanto, determinados resultados não podem ser aplicados a grupos específicos, como idosos institucionalizados, ou os adultos jovens.

Entretanto, mesmo diante de algumas limitações, os estudos são consistentes, de amostras consideráveis, e apontam para um cuidado especial, e a necessidade de avaliação e observação mais criteriosa da qualidade do sono do idoso, e suas possíveis consequências. 


\section{REFERÊNCIAS}

1. Veras R. Envelhecimento populacional contemporâneo: demandas, desafios e inovações. Rev Saud Públic 2009; 43 (3): 1-7.

2. Gassmann KG, Rupprecht R, Freiberger E. Predictors for occasional and recurrent falls in community-dwelling older people. Z Gerontol Geriat. 2009; 42: 3-10.

3. Nogal ML. Caídas em el anciano. Medicina Clinica 2009; 133 (4): 147-153.

4. Gama ZAS, Gómez-Conesa A. Factores de riesgo de caídas en ancianos: revisión sistemática. Rev Saud Públic 2008; 42(5): 946-56.

5. Ribeiro AP, Souza ER, Atie S, Souza AC, Schilithz AO. A influência das quedas na qualidade de vida de idosos. Ciênc Saúd Colet 2008; 13 (4): 1265-1273.

6. Rubenstein LZ. Falls in older people: epidemiology, risk factors and strategies for prevention. Age and ageing 2006; 35:37-41.

7. Fabrício SCC, Rodrigues RAP, Júnior MLC. Causas e consequiências de quedas de idosos atendidos em hospital público. Rev Saud Pública 2004; 38(1): 93-9.

8. Perracine MR, Ramos LR. Fatores associados a quedas em uma coorte de idosos residentes na comunidade. Rev Saud Pública 2002; 36 (6): 709-16.

9. Siqueira FV, et al. Prevalência de quedas em idosos e fatores associados. Rev Saud Pública 2007: 41(5): 749-56.

10. Gawryszewiski VP, Jorge MHPM, Koizume MS. Mortes e Internações por causas externas no Brasil: o desafio de integrar a saúde coletiva e atenção individual. Rev Assoc Med Bras. 2004; 50(1): 97-103.

11. Mazo GZ, Liposcki DB, Ananda C, Prevê D. Condições de saúde, incidência de quedas e nível de atividade física dos idosos. Rev Bras Fisioter 2007; 11 (6): 437-442.

12. Roth T. Novel outcome measures of sleep, sleep loss and insomnia. Sleep Medicine 2008; 9 (Suppl 1): 1-2.

13. Ancoli-Israel S, Ayalon L, Salzman C. Sleep in the elderly: normal variations and common sleep disorders. Harv Rev Psychiatry 2008; 16 (5): 279-286.

14. Faubel R, et al. Sleep duration and healthrelated quality of life among older adults: a population-based cohort in Spain. Med Res 2009; 32 (8): 1059-1068.
15. Fernandes RMF. O sono normal. Med Res 2006; 39 (2): 157-168.

16. Harrington JJ, Lee-Chiong Jr T. Sleep and older patients. Clin Chest Med. 2007; 28: 673-684.

17. Dregan A, Armstrong D. Age, cohort and period effects in the prevalence of sleep disturbances among older people: the impact of economic downturn. Social Science \& Medicine 2009; 69: $1432-1438$.

18. Wolkove N, Elkholy O, Baltzam M, Palayew M. Sleep and aging: sleep disorders commonly found in older people. CMJA. 2007; 176 (9): 1299-1304.

19. Ohayon MM, Carskadon MA, Guilleminaut C, Vitiello MV. Meta-analysis of quantitative sleep parameters from childhood to old age in healthy individiduals: developing normative sleep values across the human lifespan. Sleep. 2004; 27 (7): $1255-73$.

20. Xiang YT, et al. Relationships of sleep duration with sleep disturbances, basic sociodemographic factors, and BMI in Chinese people. Sleep Medicine 2009; 10: 1085 - 1089.

21. Ohayon MM, Vecchierine MF. Normative sleep data, cognitive function and daily living activities in older adults in the community. Sleep 2005; 28 (8): 981-9.

22. Fragoso CAV, Gil TM. Sleep complaints in community-living older persons: a multifactorial geriatric syndrome. JAGS 2007; 55 (11): 1853-1866.

23. Ancoli-Israel S. Sleep and aging: prevalence of disturbed sleep and treatment considerations in older adults. J Clinical Psychiatry 2005; 66 (supp1 9): 24-30.

24. Ancoli-Israel S. Sleep and its disorders in aging populations. Sleep Med 2009; 10: S7-S11.

25. Tsai Yun-Fang, Wong TKS, Ku Yan-Chiou. Selfcare management of sleep disturbances and risk factors for poor sleep among older residents of Taiwanese nursing homes. JCNR Care.2008; 1219-1226.

26. Stone KL, Ensrud KE, Ancoli-Israel S. Sleep, insomnia and falls in elderly patients. Sleep Medicine 2008; 1 (9): S18-S22.

27. American Psychiatric Association . Diagnostic and Statistical Manual of Mental Disorders .[acesso em 12 fev 2010]. Disponível em: http:www.psych.org 
28. Edinger JD, et al. Derivation of research diagnostic criteria for insomnia: report of an American Academy of Sleep Medicine work group. Sleep Med 2004; 27 (8): 1567-1596.

29. Ohayon MM. Epidemiology of insomnia: what we know and what we still need to learn. Sleep Med 2002; 6 (2): 97-111.

30. Krishnan P, Hawranik P. Diagnosis and management of geriatric insomnia: a guide for nurse practitioners. J Am Acad Nurse Practitioners 2008; 20: 590-599.

31. Misra S, Malow BA. Evaluation of sleep disturbances in older adults. Clinics in Geriatric Medicine. 2008; 24: 15-26.

32. Stone KL, et al. Self-Reported sleep nap habits and risk of falls and fractures in older women: the study of osteoporotic fractures. J American Geriatric Society 2006; 54: 1177-1183.

33. Goldman SE, et al. Poor sleep is associated with poorer physical performance and greater functional limitations in older women. Sleep 2007; 30(10): 1317-1324.

34. Stone KL, et al. Actigraphy-Measured Sleep Characteristics and Risk of Falls in Older Women. Arch Inter Med 2008; 168 (16): 17681775.
35. Dam TTL, et al. Association Between Sleep and Physical Function in Older Men: The Osteoporotic Fractures in Men Sleep Study. JAGS 2008; 56:1665-1673.

36. Endeshaw Y. Correlates of self-reported nocturia among community-dwelling older adults. J Gerontol 2009; 62A (1): 142-148.

37. Hill EL, et al. Sleep disturbances and falls in older people. J Gerontol 2007; 62 (1):62-66.

38. Kaushik S, Wang JJ, Mitchell P. Sleep apnea and falls in older people. J Am Geriatric Society 2007; 55(7):1149-50.

39. George RJ, Delbaere K, Williams P, Lord S. Sleep quality and falls in older people living in self and assisted-care villages. Gerontol 2009; 55: 162-168.

40. Teo JSH, et al . Do sleep problems or urinary incontinence predict falls in eldely women? Australian J Physiother 2006; 52: 19-24.

41. Kuo HK, et al. Gender-Specific association between self-reported sleep duration in highfunctioning older adults. J Gerontol 2009; 6(2):450-9

42. Asplund R. Nocturia in relation to sleep, health, and medical treatment in the elderly. BJU International 2005; 96(Suppl 1): 15-21 